\title{
Waves without the wave equation: Examples from nonlinear acoustics
}

\author{
Simba K. Dziwa \\ Department of Mathematics 85 Applied Mathematics, \\ University of Pretoria, South Africa. \\ Niko Sauer \\ Centre for the Advancement of Scholarship, \\ University of Pretoria, South Africa.
}

\begin{abstract}
The traditional wave equation is mostly, if not always, obtained from a system of first order partial differential equations augmented by constitutive relations. These are often nonlinear and linearizations are forcibly applied.

In a nonlinear system of first order partial differential equations the criterion for hyperbolicity, necessary for the description of wave phenomena, involves the solution. It is therefore possible that solutions may evolve in such a way that hyperbolicity is challenged in the sense that the system comes close to not being hyperbolic. We use the recently introduced formulation for nonlinear acoustic disturbances to illustrate. When hyperbolicity deteriorates, standard numerical methods and the heuristics surrounding wave motion may be compromised. To overcome such difficulties we introduce the notion of inverse characteristic which, at least in the examples, reduces numerical calculations to elementary techniques and clarifies intuition. Analysis of inverse characteristics leads to two systems of ordinary differential equations that have time-like trajectories and space-varying associated curves. Time-like trajectories give rise to an alternative measure of time in terms of which space-like trajectories are easier to analyze. Space-varying curves enable the analysis of shock phenomena in a direct way. We give conditions under which an initially mild challenge of hyperbolicity, represented by pressure, develops into a severe challenge. Under these conditions violent velocity shocks develop from an initially undisturbed state.
\end{abstract}

Keywords: Hyperbolic systems, shock phenomena, inverse characteristics, nonlinear acoustics. \footnotetext{
$E m$ (aner)
Saut

Email addresses: Simba@dziwainc.com (Simba K. Dziwa), niko.sauer@up.ac.za (Niko
} 


\section{Introduction}

In 1747 D'Alembert unveiled the wave equation in one space dimension and the general form of its solutions. To some mathematicians and those who apply mathematics, this was the beginning of a life-long romance. If one carefully scrutinizes the various derivations a number of critical points turn up. For instance, in an elastic bar longitudinal disturbances cannot be one-dimensional and paradoxical consequences are not hard to come by. After all, the bar would become thinner when stretched and thicker where it is compressed!

The equations for wave-like disturbances are, within the context of continuum mechanics, based on balance of linear momentum, conservation of mass and constitutive relations. These usually manifest in first order partial differential equations. From there ruthless procedures of elimination and linearization lead to the dearly beloved and greatly admired wave equation.

It was in 1958 that K. O. Friedrichs, in his study of positive linear systems partial differential equations, pointed out that the wave equation could be derived from a system of first order equations, thus taking a step back towards the underlying physical principles.

In a recent paper, Sauer [9], the equations of nonlinear acoustics were reformulated in terms of the so-called Lagrangian description of fluid motion (introduced by Euler). In [10] the formulation is discussed in a more succinct manner. The use of a coordinate system that follows every 'point' in a reference configuration was used to accommodate the principle of a materially closed system. Balance of momentum and conservation of mass combined into a system of first order equations. The equation of state for gas under isentropic conditions lead to the constitutive equation. Further analysis revealed that the first order system of governing partial differential equations is also subject to an inequality constraint derived from physical considerations.

For situations with constant density in the reference configuration the equations can be scaled to dimensionless form and if the motion is one dimensional in space, the differential equations are

$$
\left.\begin{array}{l}
v_{t}(t, x)+p_{x}(t, x)=0 \\
p_{t}(t, x)+[1+p(t, x)]^{2} v_{x}(t, x)=0 .
\end{array}\right\}
$$

Here $v(t, x)$ is the dimensionless velocity of "particle" $x \in \mathbb{R}$ in the reference configuration at time $t>0$ and $p(t, x)$ the dimensionless pressure experienced by it. Velocity is scaled according to "thermostatic sound speed" which then corresponds to $v=1$. It is of interest to note that this system of equations is not invariant under interchange of the variables $v$ and $p$. For systems that lead to the wave equation this invariance is essential. Brute force elimination of $v$ in (1), with many liberties taken, leads to the nonlinear wave equation

$$
p_{t t}-\frac{2 p_{t}^{2}}{1+p}-[1+p]^{2} p_{x x}=0 .
$$

It may be better to stay with (1) which is close to the original derivation. 
The constraint, expressed in the form

$$
1+p(t, x)>0,
$$

needs elucidation. If $J(x, t)$ is the Jacobian of the motion $X(t)=\Psi(x, t)$ it is shown in [9] that the principle of conservation of mass can be expressed in the form $\rho(x)=J(x, t) \sigma(X, t)$ with $\rho(x)$ the mass-density in the reference configuration and $\sigma(X, t)$ the mass density at time $t$. Hence, for densities to be positive, it is a physical requirement that $J(x, t)>0$. It is also shown that a Boyle-Mariotte relation holds in the form $[1+p(t, x)] J(x, t)=1$. The constraint is derived from the positivity of $J$. Mathematically it ensures that the system (1) is hyperbolic. In fact, the crucial eigenvalues that determine hyperbolicity are $1+p$ and $-(1+p)$. Along with this are two eigenvectors of the form $([1+p], 1)$ and $(-[1+p], 1)$ which are linearly independent. Hyperbolicity of a system is essential for it to exhibit wave-like propagation of disturbances. It is challenged when $1+p(t, x)$ approaches zero. Then the corresponding eigenvectors are nearly the same. This can cause computational difficulties. Even worse, since hyperbolicity depends on the solution, a challenge may develop as time goes on. In turn the wave-like character of solutions could come close to being lost.

An example in [9] illustrates the strange behaviour exhibited by the system when the initial pressure challenges the constraint for large $x$. The case in question is where initially $v(0, x)=0$ and $1+p(0, x)=\exp \left\{-x^{2}\right\}$. Rough, limited numerical computations of characteristics revealed the curious behaviour that the (bell-shaped) initial pressure profile decays towards the limit enforced by the constraint, so that hyperbolicity becomes severely challenged. On the other hand the gas, initially at rest, becomes increasingly unsettled as time goes on. Also, activity appeared to be confined to an interval outside of which the Gaussian curve does not contribute significantly to the occurrence of events. In the present paper we develop tools to investigate this observation in a precise manner for a wide class of initial pressures. The focus will mostly be on cases where $1+p(0, x) \rightarrow 0$ as $x \rightarrow \infty$ which means that the constraint is initially challenged "far away." The case $x \rightarrow-\infty$ is similar.

To deal with the constraint, the variable $q$ is introduced as follows:

$$
1+p(t, x)=\exp \{q(t, x)\}
$$

This transforms the system (1) into

$$
\left.\begin{array}{l}
v_{t}(t, x)+\exp \{q(t, x)\} q_{x}(t, x)=0 \\
q_{t}(t, x)+\exp \{q(t, x)\} v_{x}(t, x)=0 .
\end{array}\right\}
$$

The quasi-linear system (4) is symmetric hyperbolic (see e.g. Jeffrey [5], Lax $[8]$ ), and can be transformed to canonical form by the substitutions

$$
\left.\begin{array}{l}
u_{1}(t, x)=\frac{1}{2}[v(t, x)+q(t, x)] ; \\
u_{2}(t, x)=\frac{1}{2}[v(t, x)-q(t, x)],
\end{array}\right\}
$$


to obtain

$$
\left.\begin{array}{l}
u_{1, t}(t, x)+\exp \{q(t, x)\} u_{1, x}(t, x)=0 ; \\
u_{2, t}(t, x)-\exp \{q(t, x)\} u_{2, x}(t, x)=0 ; \\
q(t, x)=u_{1}(t, x)-u_{2}(t, x) .
\end{array}\right\}
$$

The system (6) is amenable to analysis by the method of characteristics (see e.g. Courant-Friedrichs [3, Chap II], Lax [7, 8]). Indeed, the function $u_{1}$ is constant along the curves $\mathrm{C}_{1}: x=X_{1}(t)$ and so is $u_{2}$ along the curves $\mathrm{C}_{2}: x=X_{2}(t)$ that satisfy the ordinary differential equations

$$
\left.\begin{array}{l}
X_{1}^{\prime}(t)=\exp \left\{q_{1}\right\} ; \\
X_{2}^{\prime}(t)=-\exp \left\{q_{2}\right\},
\end{array}\right\}
$$

with $q_{1}$ and $q_{2}$ the restrictions of $q$ to the curves $\mathrm{C}_{1}$ and $\mathrm{C}_{2}$. Thus $u_{1}$ and $u_{2}$ are Riemann invariants of (4). In form the system (6) suggests a "propagation speed" $\exp \{q\}=1+p$ which is pressure dependent. For the wave equation propagation speed is constant.

The initial conditions for the system $(1)$ are $v(0, x)=v_{0}(x) ; p(0, x)=$ $p_{0}(x)$, and these are, from (5) and (3), transcribed to initial conditions for the transformed system (6):

$$
\left.\begin{array}{l}
u_{10}(x)=\left[v_{0}(x)+q_{0}(x)\right] / 2 ; \\
u_{20}(x)=\left[v_{0}(x)-q_{0}(x)\right] / 2 ; \\
1+p_{0}(x)=\exp \left\{q_{0}(x)\right\} .
\end{array}\right\}
$$

Our first quest will be to reduce the system (6) under the initial conditions (8), with $v_{0}=0$ to systems of ordinary differential equations in a way that circumvents the calculation of characteristics. In Sect. 2 this is achieved by an inverse approach, namely to map a given point $(t, x)$ in the $t x$-plane to points $a_{1}(t, x)$ and $a_{2}(t, x)$ on the $x$-axis $(t=0)$ where the characteristics $\mathrm{C}_{1}$ and $\mathrm{C}_{2}$ through $(t, x)$ come from (Fig. 1).

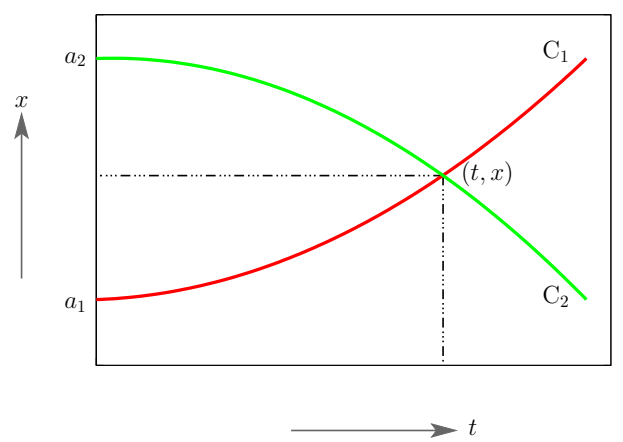

Figure 1: Inverse characteristics

We shall (humbly) refer to these functions as inverse characteristics. They not only dominate further considerations, but are indeed instrumental in the 
reduction of numerical calculations to the solution of equations of the form $F(x)=0$ and, perhaps, numerical integration. Moreover, shock phenomena turn out - as can be expected - to be associated with at least one of the inverse characteristics being multi-valued.

The work presented here mainly concentrates on the situation where the constraint is initially challenged at $x=\infty$ with $1+p_{0}(x)$ decreasing to zero as $x \rightarrow \infty$. To cope with a similar challenge at $x=-\infty$ and symmetrical situations, we derive in Sect. 3 invariance properties of the equations (1) under translations and reflections of the "directional" variables $x$ and $v$. Asymptotic behaviour of inverse characteristics for large $x$ is studied in Sect. 4 .

Section 5 is devoted to the study of a family of time-like curves ( $x$ as parameter) associated with the inverse characteristics. These curves do not necessarily cover the whole plane in question, but some of them may go on unhindered. In Sect. 6 we give two model-examples to serve as illustrations of ideas, notations and results of previous and subsequent sections.

Unrestrained time-like curves can be used as convenient measures of time as will be pointed out in Sect. 7. This convenience is used in Sect. 8 to give a method for calculating a families of space-varying curves with "new-time" as parameter. Once again we use an inverse approach which leads to very explicit results. Some of these curves may develop discontinuities which appear as zeros in the inverse view. The handling of discontinuities is treated in Sect. 9. With this in hand, calculation of solution curves of (1) becomes possible with discontinuities in the inverse characteristics directly related to shock discontinuities in pressure as well as velocity. This is further discussed in Sect.10. The interesting fact is that velocity becomes untamed at the shock, increasing above "sound speed" $(v=1)$. This was predicted by Earnshaw [2] in regard to the piston problem ([10]). In Sect. 11 it is shown that pressure does indeed decay and that the same happens to power (pressure times velocity). The results are illustrated in Sect.12. They illustrate the 'havoc created' when 'strict hyperbolicity' is deviated from (Dafermos [1, Chap. VII]), in a very real example. It turns out that the notion of confinement is a deception created in [9] by not waiting long enough for shocks to develop.

In Sect. 13 we consider two linearizations. The first of these concerns the system (1), which essentially gives the wave equation by ignoring the constraint. The second is a linearization of (4) in which a flavour of the constraint is preserved. The linearizations illustrate the essential effects of the constraint.

The method we use differs from the direct approach by means of characteristics as followed by Thomas [11] for the ideal gas equations in Eulerian form (misnamed as it was introduced by D'Alembert) and, in more general form, by Jeffrey [4]. The discussion in Courant-Friedrichs [3, Chap. III] follows a different route. 


\section{Reduction to ordinary differential equations}

We consider the Cauchy problem (6), (8), with $v_{0}(x)=0$ so that the initial conditions (8) simplify to

$$
u_{10}(x)=\frac{1}{2} q_{0}(x) ; \quad u_{20}(x)=-\frac{1}{2} q_{0}(x) .
$$

Let $(t, x)$ be a point in the $t x$-plane, and consider the characteristics passing through this point. Suppose that the characteristic $\mathrm{C}_{1}$ emanates from the point $\left(0, a_{1}(t, x)\right)$ and $\mathrm{C}_{2}$ starts at $\left(0, a_{2}(t, x)\right)$. By $(7), \mathrm{C}_{1}$ is increasing and $\mathrm{C}_{2}$ decreasing. It follows that $a_{1}<a_{2}$. Since $u_{1}$ is constant along $\mathrm{C}_{1}$ and $u_{2}$ is constant along $\mathrm{C}_{2}$, it is clear from (9) that

$$
u_{1}(t, x)=\frac{1}{2} q_{0}\left(a_{1}(t, x)\right) ; \quad u_{2}(t, x)=-\frac{1}{2} q_{0}\left(a_{2}(t, x)\right),
$$

and hence, from (5),

$$
v(t, x)=\frac{1}{2}\left[q_{0}\left(a_{1}\right)-q_{0}\left(a_{2}\right)\right] ; \quad q(t, x)=\frac{1}{2}\left[q_{0}\left(a_{1}\right)+q_{0}\left(a_{2}\right)\right] .
$$

Thus, if $a_{1}$ and $a_{2}$ can be found, the Cauchy problem (6), (9) is solved. It is of interest to note that the inverse characteristics $a_{1}, a_{2}$ satisfy the following equations as can be seen by substitution of (10) into (6):

$$
\left.\begin{array}{l}
a_{1, t}+\exp \left\{\left[\frac{1}{2}\left[q_{0}\left(a_{1}\right)+q_{0}\left(a_{2}\right)\right]\right\} a_{1, x}=0 ;\right. \\
a_{2, t}-\exp \left\{\left[\frac{1}{2}\left[q_{0}\left(a_{1}\right)+q_{0}\left(a_{2}\right)\right]\right\} a_{2, x}=0 .\right.
\end{array}\right\}
$$

We shall assume throughout that $p_{0}$ (and therefore $q_{0}$ ) is continuously differentiable.

From (5) and (10) we see also that

$$
\begin{aligned}
q_{1}(s)=q\left(s, X_{1}(s)\right)=\left[u_{1}-u_{2}\right]\left(s, X_{1}(s)\right) & \\
= & u_{10}\left(a_{1}\right)-u_{2}\left(s, X_{1}(s)\right) \\
& =\frac{1}{2} q_{0}\left(a_{1}(t, x)\right)-u_{2}\left(s, X_{1}(s)\right) .
\end{aligned}
$$

Similarly, along $\mathrm{C}_{2}$,

$$
q_{2}(s)=u_{1}\left(s, X_{2}(s)\right)+q_{0}\left(a_{2}(t, x)\right) .
$$

Integration of the equations in (7) gives, with the aid of (13), (14) and the fact that $(t, x)=\left(t, X_{1}(t)\right)=\left(t, X_{2}(t)\right)$,

$$
\begin{aligned}
& x-a_{1}(t, x)=X_{1}(t)-X_{1}(0)=\exp \left\{\frac{1}{2} q_{0}\left(a_{1}\right)\right\} \int_{0}^{t} \exp \left\{-u_{2}\left(s, X_{1}(s)\right)\right\} d s . \\
& a_{2}(t, x)-x=-\left[X_{2}(t)-X_{2}(0)\right]=\exp \left\{\frac{1}{2} q_{0}\left(a_{2}\right)\right\} \int_{0}^{t} \exp \left\{u_{1}\left(s, X_{2}(s)\right)\right\} d s .
\end{aligned}
$$


Next we differentiate (15) with respect to $t$ :

$$
\begin{aligned}
&-a_{1, t}(t, x)=\frac{1}{2} q_{0}^{\prime}\left(a_{1}\right) a_{1, t} \exp \left\{\frac{1}{2} q_{0}\left(a_{1}\right)\right\} \int_{0}^{t} \exp \left\{-u_{2}\left(s, X_{1}(s)\right)\right\} d s \\
&+\exp \left\{q_{0}\left(a_{1}\right)-u_{2}\left(t, X_{1}(t)\right\}\right. \\
&=\left[\frac{1}{2}\left(x-a_{1}\right) q_{0}^{\prime}\left(a_{1}\right)\right] a_{1, t}+\exp \left\{\frac{1}{2}\left[q_{0}\left(a_{1}\right)+q_{0}\left(a_{2}\right)\right\} .\right.
\end{aligned}
$$

Re-arrangement of (17) yields the expression

$$
\left[1+\frac{1}{2}\left(x-a_{1}\right) q_{0}^{\prime}\left(a_{1}\right)\right] a_{1, t}=-\exp \left\{\frac{1}{2}\left[q_{0}\left(a_{1}\right)+q_{0}\left(a_{2}\right)\right]\right\} .
$$

A similar treatment of (16) leads to

$$
\left[1-\frac{1}{2}\left(a_{2}-x\right) q_{0}^{\prime}\left(a_{2}\right)\right] a_{2, t}=\exp \left\{\frac{1}{2}\left[q_{0}\left(a_{1}\right)+q_{0}\left(a_{2}\right)\right]\right\} .
$$

For fixed $x$ the equations (18), (19) is a system of ordinary differential equations with initial condition

$$
a_{1}(0, x)=x ; \quad a_{2}(0, x)=x,
$$

which, at least for small $t$, can be solved uniquely for the much sought after $a_{1}$ and $a_{2}$. Thereby hangs a tale.

If there is more than one solution (for larger $t$ ) there would be a point $(t, x)$ through which at least two $\mathrm{C}_{1}$ or $\mathrm{C}_{2}$ - characteristics pass. This is when shock phenomena occur.

A cursory glance at (15) and (16) shows that $a_{1}(t, x) \leq x \leq a_{2}(t, x)$. Hence the signature of the left-most terms in (18) and (19) depends very much on the behaviour of the derivative $q_{0}^{\prime}$. With this in mind, we define two functions:

$$
\left.\begin{array}{l}
f_{1}(z ; x)=1+\frac{1}{2}(x-z) q_{0}^{\prime}(z) ; z \leq x \\
f_{2}(z ; x)=1-\frac{1}{2}(z-x) q_{0}^{\prime}(z) ; z \geq x,
\end{array}\right\}
$$

so that the equations (18) (19) may be expressed in the form

$$
\left.\begin{array}{l}
f_{1}\left(a_{1}, x\right) a_{1, t}=-\exp \left\{\frac{1}{2}\left[q_{0}\left(a_{1}\right)+q_{0}\left(a_{2}\right)\right]\right\} ; \\
f_{2}\left(a_{2}, x\right) a_{2, t}=\exp \left\{\frac{1}{2}\left[q_{0}\left(a_{1}\right)+q_{0}\left(a_{2}\right)\right]\right\} .
\end{array}\right\}
$$

We may also use the identities (15), (16) to find suitable expressions for spatial derivatives. Indeed, differentiation with respect to $x$ gives

$$
\left.\begin{array}{l}
f_{1}\left(a_{1}, x\right) a_{1, x}=1 ; \\
f_{2}\left(a_{2}, x\right) a_{2, x}=1 .
\end{array}\right\}
$$

These expressions, which are uncoupled, show that singularities in $a_{1}$ or $a_{2}$ may develop at zeros of $f_{1}$ and $f_{2}$. We also note that the expressions (22), (23) are in complete accord with (12). 


\section{Translations and reflections}

Invariance under certain substitutions are useful for the transfer of results obtained under apparently restrictive assumptions and the understanding of the nature of the equations we deal with. These are translations and reflections. New variables will be denoted by asterisks.

By translation we mean the change of variable

$$
x^{*}=x-x_{0} ; \quad v^{*}\left(t, x^{*}\right)=v\left(t, x-x_{0}\right) ; \quad p^{*}\left(t, x^{*}\right)=p\left(t, x-x_{0}\right) .
$$

Reflection affects the variables $x, v$ and $p$ differently. It is defined by

$$
x^{*}=-x ; \quad v^{*}\left(t, x^{*}\right)=-v(t,-x) ; \quad p^{*}\left(t, x^{*}\right)=p(t,-x) .
$$

The following result is proved by direct substitution into (1), (5), (6) and (12).

Theorem 1. Under the translation (24) and the reflection (25) the system of equations (1) and the constraint (2) retain their form. Under reflection the following holds:

$$
\left.\begin{array}{ll}
u_{1}^{*}\left(t, x^{*}\right)=-u_{2}(t,-x) ; & u_{2}^{*}\left(t, x^{*}\right)=-u_{1}(t,-x) . \\
a_{1}^{*}\left(t, x^{*}\right)=-a_{2}(t,-x) ; & a_{2}^{*}\left(t, x^{*}\right)=-a_{1}(t,-x),
\end{array}\right\}
$$

where $u_{k}^{*}$ and $a_{k}^{*}$ are derived from transformed quantities as in Sect.2.

\section{Asymptotic behaviour}

Important for our considerations is the asymptotic behaviour of the functions $a_{1}(t, x)$ and $a_{2}(t, x)$ for large $x$ with $t$ fixed. From the definitions (21) we see directly that the curves $a_{1}(t, x)=x$ and $a_{2}(t, x)=x$ are solutions of the differential equations (23). One may ask: when will these curves "attract" solutions of the equations in question? Towards an answer we re-phrase the expressions (15), (16) purely in terms of $p_{0}$.

Let us first consider the case of $a_{1}$ by noticing that, from (10), $\exp \left\{-u_{2}\left(s, X_{1}(s)\right)\right\}=\frac{1}{2} q_{0}\left(a_{2}\left(s, X_{1}(s)\right)\right.$. The definition (3) now leads from (15) to

$$
x-a_{1}(t, x)=\left[1+p_{0}\left(a_{1}\right)\right]^{1 / 2} \int_{0}^{t}\left[1+p_{0}\left(a_{2}\left(s, X_{1}(s)\right)\right)\right]^{1 / 2} d s .
$$

Similarly, the expression (16) can be re-written in the form

$$
a_{2}(t, x)-x=\left[1+p_{0}\left(a_{2}\right)\right]^{1 / 2} \int_{0}^{t}\left[1+p_{0}\left(a_{1}\left(s, X_{2}(s)\right)\right)\right]^{1 / 2} d s .
$$

Theorem 2. Suppose there are positive constants $M$ and $N$ such that for all $x$

$$
1+p_{0}(x) \leq M
$$


and

$$
\left|x p_{0}^{\prime}(x)\right| \leq N .
$$

Then, for fixed $t$,

$$
\left.\begin{array}{l}
a_{1}(t, x) \sim x-\left[1+p_{0}(x)\right] t ; \\
a_{2}(t, x) \sim x+\left[1+p_{0}(x)\right] t,
\end{array}\right\}
$$

as $x \rightarrow \pm \infty$.

Proof. We deal with case $x \rightarrow \infty$. The other case can be dealt with analogously, but would simply follow from Theorem 1 .

Thanks to (26) and (27), it follows from (28) that

$$
\left.\begin{array}{l}
0 \leq x-a_{1}(t, x) \leq M t \\
0 \leq a_{2}(t, x)-x \leq M t
\end{array}\right\}
$$

It immediately follows from (31) that, as $x \rightarrow \infty$,

$$
\left.\begin{array}{l}
\vartheta_{1}:=\frac{a_{1}}{x} \rightarrow 1 ; \\
\vartheta_{2}:=\frac{a_{2}}{x} \rightarrow 1 .
\end{array}\right\}
$$

Now, let us examine the integrand in the expression (26). Since the $\mathrm{C}_{1}$ characteristics are increasing and the $\mathrm{C}_{2}$-family decreasing, we see that

$$
\left.\begin{array}{r}
\vartheta_{1} \leq \frac{X_{1}(s)}{x} \leq \vartheta_{2} ; \\
\vartheta_{1} \leq \vartheta:=\frac{a_{2}\left(s, X_{1}(s)\right.}{x} \leq \vartheta_{2},
\end{array}\right\}
$$

for $0 \leq s \leq t$. Thus $X_{1}(s) / x \rightarrow 1$ and $\vartheta \rightarrow 1$ uniformly in $s \in[0, t]$ when $x \rightarrow \infty$. Next we employ the mean value theorem to obtain

$$
p_{0}\left(a_{2}\left(s, X_{1}(s)\right)-p_{0}(x)=[\vartheta-1]\left(\frac{x}{\xi}\right) \xi p_{0}^{\prime}(\xi)\right.
$$

with either $a_{2}\left(s, X_{1}(s)\right)<\xi<x$ or $x<\xi<a_{2}\left(s, X_{1}(s)\right)$. Combination of (29) and (33) yields

$$
\mid p_{0}\left(a_{2}\left(s, X_{1}(s)\right)-p_{0}(x)|\leq N| \vartheta-1 \mid\left(\frac{x}{\xi}\right) .\right.
$$

We also see from (32) that either $x / \xi \leq 1 / \vartheta_{1}$ or $x / \xi<1$. Hence it follows from (34) that $p_{0}\left(a_{2}\left(s, X_{1}\right)\right) \rightarrow p_{0}(x)$ uniformly in $s$ as $x \rightarrow \infty$. Therefore the integral in (26) converges to $t\left[1+p_{0}(x)\right]^{1 / 2}$ as $x \rightarrow \infty$. In a similar (but less excruciating) way it is proved that $1+p_{0}\left(a_{1}(t, x)\right) \rightarrow 1+p_{0}(x)$ as $x \rightarrow \infty$. With that the first of the asymptotic expressions in (30) is verified. The second one follows similarly. 
Theorem 2 shows that under the assumptions (28), (29) wave-like behaviour is exhibited for large $x$ with $a_{1}$ representing downstream and $a_{2}$ upstream motion. The speed of propagation in such cases is $1+p_{0}(x)$.

Let us also remark that the uniform upper bound (28) is in accord with the placement condition, Dafermos [1, Chap. II, p.27], expressed in the form $J(x, t) \geq 1 / M>0$ for some positive constant $M$. As remarked in Sect. 1, the Boyle-Mariotte relation $([1+p(t, x)] J(x, t)=1)$ at least at time $t=0$ now implies the uniform upper bound. Although not always necessary, this bound will be assumed throughout.

\section{Time-like curves}

As can be seen from the representation (10), the role of the functions $a_{1}$ and $a_{2}$ is to define a coordinate system from which solutions of the Cauchy problem can be read off. We begin by defining time-like curves in the $a_{1} a_{2}$-plane as the trajectories of the solutions of the system of ordinary differential equations (18), (19) under the initial conditions (20), with $x$ as parameter. Within the $a_{1} a_{2}{ }^{-}$ coordinate system the trajectories describe flow lines followed by the various "particles" $x$.

For easier handling we make the substitution

$$
b_{1}(t, x)=x-a_{1}(t, x) ; \quad b_{2}(t, x)=a_{2}(t, x)-x,
$$

and immediately note that the functions $b_{1}$ and $b_{2}$ should be non-negative as can be seen from (15) and (16). The differential equations (18), (19) may now be combined and expressed as follows:

$$
\left[1+\frac{1}{2} b_{1} q_{0}^{\prime}\left(x-b_{1}\right)\right] b_{1, t}=\left[1-\frac{1}{2} b_{2} q_{0}^{\prime}\left(x+b_{2}\right)\right] b_{2, t},
$$

which links $b_{1}$ and $b_{2}$. The initial condition, derived from (20) and (35), is

$$
b_{1}(0, x)=b_{2}(0, x)=0 .
$$

Instead of leaving the problem (36), (37) to the mercy of intricate numerical methods, we take it one step further by integrating over the interval $[0, t]$. The result is:

$$
b_{1}(t, x)+\frac{1}{2} \int_{0}^{t} b_{1} q_{0}^{\prime}\left(x-b_{1}\right) b_{1, s} d s=b_{2}(t, x)-\frac{1}{2} \int_{0}^{t} b_{2} q_{0}^{\prime}\left(x+b_{2}\right) b_{2, s} d s .
$$

Substitutions of the form $\sigma=b_{1}(s, x)$ and $\sigma=b_{2}(s, x)$ in (38) lead to the following relation between $b_{1}$ and $b_{2}$ :

$$
\begin{aligned}
I_{1}\left(b_{1}, x\right):=\int_{0}^{b_{1}} & {\left[1+\frac{1}{2} \sigma q_{0}^{\prime}(x-\sigma)\right] d \sigma } \\
& =\int_{0}^{b_{2}}\left[1-\frac{1}{2} \sigma q_{0}^{\prime}(x+\sigma)\right] d \sigma=: I_{2}\left(b_{2}, x\right) .
\end{aligned}
$$


More explicitly, after integration by parts, this can be expressed in the form

$$
\begin{aligned}
b_{1}-\frac{1}{2} b_{1} q_{0}\left(x-b_{1}\right)+\frac{1}{2} & \int_{0}^{b_{1}} q_{0}(x-\sigma) d \sigma \\
& =b_{2}-\frac{1}{2} b_{2} q_{0}\left(x+b_{2}\right)+\frac{1}{2} \int_{0}^{b_{2}} q_{0}(x+\sigma) d \sigma .
\end{aligned}
$$

This equation is amenable to elementary numerical methods as long as the substitutions are valid. Indeed, to calculate the time-like curve associated with the parameter $x$, one could specify a value of $a_{1}$, find $b_{1}=x-a_{1}$, calculate the left of (40), solve for $b_{2}$ and then find $a_{2}=b_{2}+x$. The road just mapped may have dangerous bends.

Suppose, for example, that $p_{0}$ is decreasing so that $q_{0}^{\prime} \leq 0$. Then the right of (39) and therefore of (40), is increasing. On the other hand, the left sides although positive for $b_{1}$ near zero, may become negative or multi-valued. In such cases the procedure fails, yielding no solution or multiple solutions. However, if $b_{1}(t, x)$ is close to zero, this cannot happen.

One avenue of exploring this phenomenon of death and resurrection is to look for envelopes of trajectories. With this in mind we re-formulate the equation (40) in terms of $a_{1}$ and $a_{2}$ to obtain

$$
\begin{aligned}
K\left(a_{1}, a_{2} ; x\right):=\left(a_{1}-x\right)[1 & \left.-\frac{1}{2} q_{0}\left(a_{1}\right)\right]+\left(a_{2}-x\right)\left[1-\frac{1}{2} q_{0}\left(a_{2}\right)\right] \\
+ & \frac{1}{2}\left[\int_{x}^{a_{1}} q_{0}(\sigma) d \sigma+\int_{x}^{a_{2}} q_{0}(\sigma) d \sigma\right]=0 .
\end{aligned}
$$

For an envelope we need the additional equation $K_{x}=0$ which amounts to

$$
\frac{1}{2}\left[q_{0}\left(a_{1}\right)+q_{0}\left(a_{2}\right)\right]-q_{0}(x)-2=0 .
$$

If it is possible to eliminate $x$ from the equations (41), (42) an envelope will indeed exist.

\section{Model-examples}

Before going any further, let us consider two examples to illustrate the discussions above and the ones that follow. The examples we use is the so-called one-sided challenge in which $p_{0}$ is a non-increasing function and the two-sided challenge where $p_{0}$ is bell-shaped - the situation touched upon in [9].

Example 1. The ONE-SIDED ChALLENGE. Let $1+p_{0}(x)=\left\{\exp \left\{-x^{2}\right\}\right.$ if $x>$ $0 ; 1$ elsewhere $\}$, so that $q_{0}(x)=\left\{-x^{2}\right.$ if $x>0 ; 0$ if $\left.x \leq 0 ;\right\}$ For this special case the integrals in (39) or (40) can be evaluated explicitly. It turns out that the integral $I_{2}$ is increasing and positive for all $x>0$. For $x \leq 2$, however, the integral $I_{1}$ on the left is an increasing function of $b_{1}$ and for larger values of $x$ it has two local extremes, even becoming negative in places. Thus unique solutions, multiple solutions and no solutions can occur. 
Example 2. The TWO-Sided Challenge. Let $1+p_{0}(x)=\exp \left\{-x^{2}\right\}$. Once again, the integrals $I_{1}$ and $I_{2}$ can be found explicitly and the result is more digestible than in Ex. 1. In fact, $I_{1}\left(b_{1}, x\right)=b_{1}\left[1-x b_{1} / 2+b_{1}^{2} / 3\right]$ and $I_{2}\left(b_{2}, x\right)=$ $b_{2}\left[1+x b_{2} / 2+b_{2}^{2} / 3\right]$. The difficulties alluded to in the first example now become clearer. The integral $I_{2}$ is, for $x>0$, strictly increasing in $b_{2}$ while for $I_{1}$ everything is fine for $0 \leq x<\sqrt{48} / 3=2.309 \ldots$ For larger $\mathrm{x}$ multiple solutions and even no solutions occur.

In both examples the hypotheses of Theorem 2 are fulfilled with $M=1$ and $N=2 / e=0.7357 \ldots$ Thus, in the one-sided case $a_{1}(t, x) \sim x$ as $x \rightarrow \infty$ and $\sim x-t$ as $x \rightarrow-\infty$, for example. In the two-sided case, $a_{1} \sim x$ as $x \rightarrow \pm \infty$. The corresponding asymptotic expressions for $a_{2}$ are derived from (30). Phase portraits for the examples are shown in Fig. 2 below. Trajectories for $x>0$ are to the right of the curve corresponding to $x=0$. Broken trajectories can clearly be seen. The disappearing part corresponds to situations where (39) (or (40)) cannot be solved. Parametric expressions for envelopes to the trajectories can, however, be found. The envelopes demarcate regions in $a_{1} a_{2}$-space that cannot be visited. In Sect. 9 this will become clear in a different way.
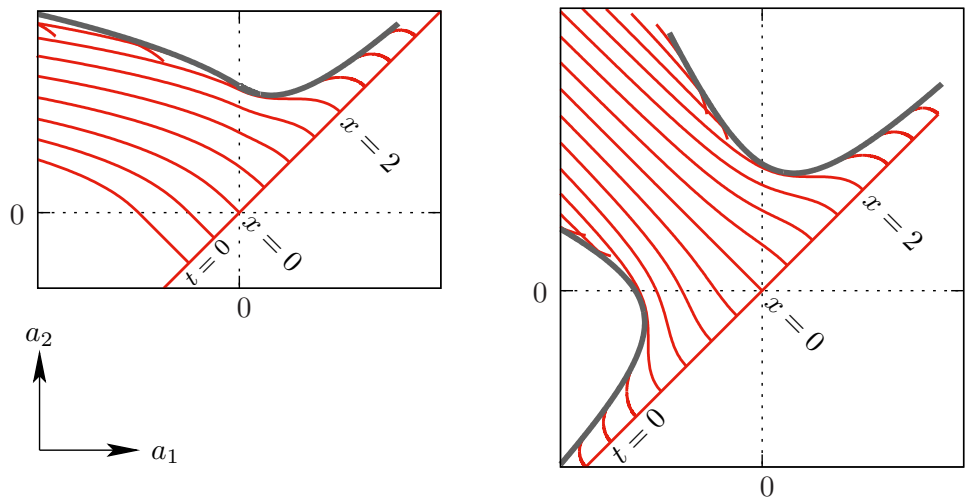

Figure 2: Time-like curves (with envelopes). Left, Ex. 1. Right, Ex. 2

We note that in Ex. 1 the input $p_{0}$ is non-increasing. In such cases the breaking up of trajectories is due to the behaviour of $I_{1}$. Non-decreasing inputs can be handled by reflection (Theorem 1) in which case the roles of $a_{1}$ and $a_{2}$ are reversed. On the other hand, Ex. 2 represents an input with increasing and decreasing parts. In this case the invariance theorem allows us to compute the two parts separately.

\section{Another measure of time}

It is convenient for us to measure time along an appropriate time-like curve. That should be an unbroken curve that extends to infinity. Such a curve will 
exist if for some $x_{0}$ the equations $1+\frac{1}{2} \sigma q_{0}^{\prime}\left(x_{0}-\sigma\right)=0$ and $1-\frac{1}{2} \sigma q_{0}^{\prime}\left(x_{0}+\right.$ $\sigma)=0$ have no positive solutions. If that is the case we can, by virtue of Theorem 1 , take $x_{0}=0$. Fig. 2 demonstrates that in the examples above, many such possibilities exist. Indeed, for both examples in question the trajectory corresponding to $x_{0}=0$ is appropriate.

Points on the chosen curve are (in $a_{1} a_{2}$-space) of the form

$$
T(t)=\left(a_{1}(t, 0), a_{2}(t, 0)\right)=\left(-b_{1}(t, 0), b_{2}(t, 0)\right):=\left(-\tau_{1}(t), \tau_{2}(t)\right) .
$$

Equation (39) establishes the following relation between $\tau_{1}$ and $\tau_{2}$. Indeed, at $x=0$,

$$
\int_{0}^{\tau_{1}}\left[1+\frac{1}{2} \sigma q_{0}^{\prime}(-\sigma)\right] d \sigma=\int_{0}^{\tau_{2}}\left[1-\frac{1}{2} \sigma q_{0}^{\prime}(\sigma)\right] d \sigma .
$$

The curve $T(t)$ in the $a_{1} a_{2}$-plane is related to time in an explicit way. Let us use the abbreviations $h_{1}(\sigma):=1+\frac{1}{2} \sigma q_{0}^{\prime}(-\sigma)=0 ; h_{2}(\sigma):=1-\frac{1}{2} \sigma q_{0}^{\prime}(\sigma)$. From the differential equations (18), (19) we have

$$
\left.\begin{array}{l}
h_{1}\left(\tau_{1}(t)\right) \tau_{1}^{\prime}(t)=\exp \left\{\frac{1}{2}\left[q_{0}\left(-\tau_{1}\right)+q_{0}\left(\tau_{2}\right)\right]\right\} ; \\
h_{2}\left(\tau_{2}(t)\right) \tau_{2}^{\prime}(t)=\exp \left\{\frac{1}{2}\left[q_{0}\left(-\tau_{1}\right)+q_{0}\left(\tau_{2}\right)\right]\right\} .
\end{array}\right\}
$$

Since $\tau_{2}$ can be expressed in terms of $\tau_{1}$ by (43), the first equation in (44) can (in principle) be integrated to obtain $t$ in terms of $\tau_{1}$ under the initial condition $\tau_{1}(0)=0$ derived from (37). In the sections that follow we shall use the parameter $\tau=\tau_{1}$ instead of time $(t)$. The effect will be fortuitous despite the "time warp" we have introduced. As a firmer grounding we have:

Theorem 3. Suppose that the equations $h_{1}(\sigma)=0$ and $h_{2}(\sigma)=0$ have no positive solutions. Then the equation (43) establishes a bi-unique relationship between $\tau_{1}$ and $\tau_{2}$. Moreover, $\tau_{1}(t)$ and $\tau_{2}(t)$ are unbounded increasing functions. If $p_{0}$ is an even function, $\tau_{1}=\tau_{2}$.

Proof. The functions $h_{1}$ and $h_{2}$ are the integrands in (43). By hypothesis they are positive for $\sigma \geq 0$. Hence the integrals in (43) are increasing functions of $\tau_{1}$ and $\tau_{2}$. Therefore the relation between $\tau_{1}$ and $\tau_{2}$ is bi-unique.

From the first of the equations (44) it is seen that $\tau_{1}$ strictly increases with $t$. If $\tau_{1}^{*}=\sup _{t>0} \tau_{1}(t)$ is finite, and $\tau_{2}^{*}$ corresponds to $\tau_{1}^{*}$, then $\lim _{t \rightarrow \infty} \tau_{1}^{\prime}(t)=$ $\exp \left\{\frac{1}{2}\left[q_{0}\left(-\tau_{1}^{*}\right)+q_{0}\left(\tau_{2}^{*}\right)\right]\right\} / h_{1}\left(\tau_{1}^{*}\right)>0$. But this limit should be zero. For $\tau_{2}$ the result follows from the second of the equations (44).

If $p_{0}$ is even, so is $q_{0}$ and $q_{0}^{\prime}$ is odd. It follows that $h_{2}(-\sigma)=h_{1}(\sigma)$ and we conclude that $\tau_{1}=\tau_{2}$.

The hypotheses of Theorem 3 are satisfied in Exs 1 and 2. In Ex. 2 the function $p_{0}$ is even and therefore $\tau=\tau_{1}=\tau_{2}$. This can also be seen in Fig. 2. It is noteworthy that in the case of Ex. 2 time $t$ can be expressed explicitly 
in terms of $\tau$. Indeed, in the first of the equations (44) $\tau_{1}=\tau_{2}=\tau$ and the resulting equation can be solved to obtain

$$
t=\frac{1}{2}\left[\int_{0}^{\tau} \exp \left\{\sigma^{2}\right\} d \sigma+\tau \exp \left\{\tau^{2}\right\}\right]
$$

with the famous Dawson integral appearing in its original form. In this case $t$ increases spectacularly with $\tau$.

The existence of appropriate "new times" $\left(\tau_{1}, \tau_{2}\right)$ will henceforth be taken for granted.

\section{Space-varying curves}

To complete the picture we study curves in the $a_{1} x, a_{2} x$-planes along which $t$ is fixed. These will be referred to as space-varying curves. We proceed to derive general equations from which they can be computed. Our point of departure is the equations (23) and we consider the equation for $a_{1}$ first. This can be expanded in the form

$$
\left[a_{1, x}\right]^{-1}=f_{1}\left(a_{1}, x\right)=1+\frac{1}{2}\left(x-a_{1}\right) q_{0}^{\prime}\left(a_{1}\right) .
$$

At least when $a_{1}$ is near $x$-which is so for small $t$ - the derivative $a_{1, x}$ is positive and $x$ can be expressed as a function of $a_{1}$. With abuse of notation, we write $x=x\left(a_{1}\right)$. The expression (45) can now be re-written in the form

$$
\frac{d}{d a_{1}}\left[x\left(a_{1}\right)-a_{1}\right]=\frac{1}{2}\left(x-a_{1}\right) q_{0}^{\prime}\left(a_{1}\right) .
$$

If the solution curve passes through a given point $a_{1}=A_{1}, x=X$, that is, $x\left(A_{1}\right)=X$, the solution of $(46)$ is

$$
x\left(a_{1}\right)=a_{1}+\left(X-A_{1}\right)\left[\exp \left\{\frac{1}{2}\left[q_{0}\left(a_{1}\right)-q_{0}\left(A_{1}\right)\right]\right\}\right] .
$$

The solution (47) can be elegantly expressed in terms of the initial pressure $p_{0}$ by use of the definition (3) and the notation

$$
P_{0}=\left[1+p_{0}\right]^{1 / 2}=\exp \left\{\frac{1}{2} q_{0}\right\},
$$

to obtain

$$
x\left(a_{1}\right)=a_{1}+\left[\frac{X-A_{1}}{P_{0}\left(A_{1}\right)}\right] P_{0}\left(a_{1}\right) .
$$

We now take recourse to the alternative measure of time. In accordance with Sect. 7 we shall assume that the time-defining curve corresponding to $x=0$ exists and use $\tau=\tau_{1}$ instead of $t$. Then, $a_{1}(t, 0)=-\tau_{1}(t)$ and $a_{2}(t, 0)=$ $\tau_{2}(t)$. Thus, by letting $\tau$ run through different values, space-varying curves can potentially be computed. Every curve will be associated with a fixed $\tau$ which 
in turn can be associated with a fixed time $t$ calculated from (44). In this case we therefore have $A_{1}=-\tau$ and $X=0$ so that the expression (49) reduces to

$$
x\left(a_{1}\right)=a_{1}+\left[\frac{\tau}{P_{0}(-\tau)}\right] P_{0}\left(a_{1}\right) .
$$

For $a_{2}$ the same procedure can be followed with (45) replaced by

$$
\frac{d x}{d a_{2}}=f_{2}\left(a_{2} ; x\right)=1-\frac{1}{2}\left(a_{2}-x\right) q_{0}^{\prime}(z)
$$

The result, after solving (51), is

$$
x\left(a_{2}\right)=a_{2}-\left[\frac{\tau_{2}}{P_{0}\left(\tau_{2}\right)}\right] P_{0}\left(a_{2}\right) .
$$

An immediate consequence is that for $\tau=0$, that is for $t=0, a_{1}=a_{2}=x$. This is graphically seen in Fig. 2. The expressions (50), (52) also present the possibility of calculating $a_{1}$ and $a_{2}$ when $x$ and $\tau$ are given. In further considerations we shall use the notation $x=x\left(a_{1}\right), x=x\left(a_{2}\right)$ for the spacevarying curves we found. If dependence on the parameter $\tau$ is to be indicated, the notation $x=x\left(a_{1} ; \tau\right), x\left(a_{2} ; \tau\right)$ will be used.

\section{Discontinuities}

Discontinuities of the functions $x \rightarrow a_{1}(t, x)$ and $x \rightarrow a_{2}(t, x)$ with $t$ fixed, are to be expected. The expressions in (23) indeed show that if either $f_{1}\left(a_{1}, x\right)$ or $f_{2}\left(a_{2}, x\right)$ has a zero, $a_{1}$ or $a_{2}$ will be discontinuous. These discontinuities correspond to local maxima/minima of the space-varying curves found in Sect. 8 . If $q_{0}$ is not increasing (as in Ex. 1) $f_{2}\left(a_{2}, x\right)$ cannot be zero, and the discontinuity will therefore occur only in $a_{1}$ since $f_{1}$ then has all the potential of reaching zero. In fact, at a point of singularity $\left(a_{1}, x\right)$ (where $f_{1}$ has a zero) we have the relation

$$
x=a_{1}-2 / q_{0}^{\prime}\left(a_{1}\right) .
$$

This describes a curve of singularity of $a_{1}$. The relation (53) can, from (48), be expressed in terms of the initial pressure:

$$
x=S\left(a_{1}\right):=a_{1}-\frac{P_{0}\left(a_{1}\right)}{P_{0}^{\prime}\left(a_{1}\right)} .
$$

We shall study discontinuities under the following additional assumptions:

A. The initial pressure $p_{0}$ is strictly decreasing over an interval $I=\left(A_{1}^{\dagger}, \infty\right)$ and $\lim _{a_{1} \rightarrow A_{1}^{\dagger}} S\left(a_{1}\right)=\infty$.

B. The function $S$ is strictly convex on $I$. 

C. For every $\tau>0, \tau P_{0}^{\prime}(-\tau)+P_{0}(-\tau)>0$.
D. $p_{0}$ is twice differentiable on $I$.

It should be noted that in Exs 1 and 2 the assumptions above are fulfilled. Indeed, $I=(0, \infty)$ and $S\left(a_{1}\right)=a_{1}+1 / a_{1}$. Some of the consequences of Assumptions A $-\mathrm{D}$ are collected in the following propositions:

Proposition 4. Under Assumptions $\mathrm{A}$ and $\mathrm{B}$ the function $P_{0}$ is strictly decreasing, $\lim _{a_{1} \rightarrow \infty} S\left(a_{1}\right)=\infty$ and the function $S$ has a unique minimum in $I$.

Let $\mathrm{H}=\left\{\left(a_{1}, x\right): S\left(a_{1}\right) \leq x\right\}$ be the closed convex region above or on the curve of singularity $x=S\left(a_{1}\right)$ which we denote by S. Next we investigate the possibility of the curve $x=x\left(a_{1}\right)$ traversing $\mathrm{H}$. This we shall do by reverting to a suitable time-like curve as in Sect. 7 and Sect. 8. If the curve $x=x\left(a_{1}\right)$ meets $\mathrm{S}$ in the point $\left(a_{1}, x\right)$, then

$$
-P_{0}^{\prime}\left(a_{1}\right)=\frac{P_{0}(-\tau)}{\tau}=: Q(\tau)
$$

as can be seen by elimination of $x$ from (54) by using (50).

Proposition 5. For $a_{1} \in I$, the point $\left(a_{1}, x\left(a_{1}\right)\right)$ is on the curve $S$ if and only if $x^{\prime}\left(a_{1}\right)=0$. It is interior to $H$ if and only if $x^{\prime}\left(a_{1}\right)<0$, outside $H$ if and only if $x^{\prime}\left(a_{1}\right)>0$.

Proof. From (46) and (48) we see that $x^{\prime}\left(a_{1}\right)=1+\left(x-a_{1}\right) q_{0}^{\prime}\left(a_{1}\right) / 2=$ $1+\left(x-a_{1}\right) P_{0}^{\prime}\left(a_{1}\right) / P_{0}\left(a_{1}\right)<0$. This, combined with (54), leads to the expression $x^{\prime}\left(a_{1}\right)=\left[S\left(a_{1}\right)-x\right] /\left[S\left(a_{1}\right)-a_{1}\right]$. As we have seen before, $S\left(a_{1}\right)>a_{1}$ on $I$ and everything follows.

Proposition 6. For fixed $\tau>0, x\left(a_{1} ; \tau\right) \rightarrow \infty$ as $a_{1} \rightarrow \infty$. For fixed $a_{1} \in I$ the function $\tau \rightarrow x\left(a_{1} ; \tau\right)$ is increasing. Moreover, $x\left(a_{1} ; \tau\right) \rightarrow \infty$ when $\tau \rightarrow \infty$.

Proof. From (52) we see that $x\left(a_{1}, \tau\right)>a_{1}$ and the first assertion is proved.

We next show that the function $\tau \rightarrow \tau / P_{0}(-\tau)=1 / Q(\tau)$ is increasing. Indeed, $Q^{\prime}(\tau)=-\left[\tau P_{0}^{\prime}(-\tau)+P_{0}(-\tau)\right] / \tau^{2}<0$ by Assumption C. Now, since $1+p_{0}$ is bounded, we have from $(50), x\left(a_{1} ; \tau\right)=a_{1}+\left[\tau / P_{0}(-\tau)\right] P_{0}\left(a_{1}\right) \geq$ $a_{1}+\left[\tau / M^{1 / 2}\right] P_{0}\left(a_{1}\right)$, which gives the required result.

This means that if $\tau^{*}>\tau$ the space-varying curve $x=x\left(a_{1} ; \tau^{*}\right)$ lies above the curve $x=x\left(a_{1} ; \tau\right)$ and there are infinitely many space-varying curves stacked upon each other.

Theorem 7. Let $\left(A_{1}^{0}, X^{0}\right)$ be the minimum point of the curve of singularity $S$. This is a point of inflection of the curve $x=P_{0}\left(a_{1}\right)$. If the curve $x=x\left(a_{1} ; \tau\right)$ passes through this point, it also inflects there. There is a unique $\tau=\tau_{c}>0$ that fits the role. 
Proof. By Assumption D differentiation of (54) is permitted. Indeed, $S^{\prime}\left(a_{1}\right)=$ $P_{0}\left(a_{1}\right) P_{0}^{\prime \prime}\left(a_{1}\right) /\left[P_{0}^{\prime}\left(a_{1}\right)\right]^{2}$. Since $S^{\prime}\left(a_{1}\right)<0$ for $a_{1} \in\left(A_{1}^{\dagger}, A_{1}^{0}\right)$ it follows that $P_{0}^{\prime \prime}\left(a_{1}\right)<0$ for such $a_{1}$. To the right of $A_{1}^{0}$ it follows in a similar manner that $P_{0}^{\prime \prime}\left(a_{1}\right)>0$. Thus $P_{0}$ is concave on $\left(A_{1}^{\dagger}, A_{1}^{0}\right)$ and convex on $\left(A_{1}^{0}, \infty\right)$. Therefore $\left(A_{1}^{0}, X^{0}\right)$ is a point of inflection of $P_{0}$.

If $X^{0}=x\left(A_{1}^{0} ; \tau\right)$ it follows from Proposition 5 that $x^{\prime}\left(A_{1}^{0} ; \tau\right)=0$. From $(50)$ we see that $x^{\prime \prime}\left(a_{1} ; \tau\right)=\left[\tau / P_{0}(-\tau)\right] P_{0}^{\prime \prime}\left(a_{1}\right)$. Thus $x\left(a_{1} ; \tau\right)$ is also concave to the left and convex to the right of $A_{1}^{0}$.

By Proposition $6 x\left(A_{1}^{0} ; \tau\right)$ increases indefinitely from $A_{1}^{0}=x\left(A_{1}^{0} ; 0\right)<X^{0}$. For some $\tau=\tau_{c}>0$ it must reach the value $X^{0}$. This value of $\tau$ is evidently unique.

The results of this section are illustrated in Fig. 3 where notations are also explained. The illustration is based on Ex. 1 where $\tau_{c}=e^{1 / 2}=1.6487 \ldots$, $A_{1}^{0}=1$ and $X^{0}=2$. The curves $x=x\left(a_{1}\right)$, shown above the diagonal line $\tau=0$ exhibit the strange "attraction" to the region $\mathrm{H}$ (Hades). The curves $x=x\left(a_{2}\right)$, corresponding to the same $\tau$-values, are shown below the diagonal. Since $f_{2}>0$, they are oblivious of $\mathrm{H}$.

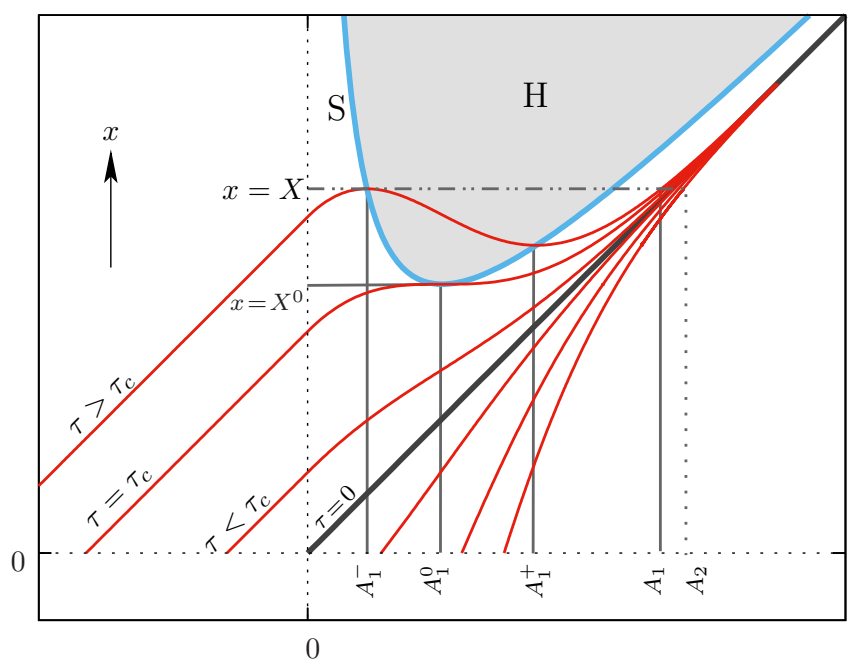

Figure 3: Space-varying curves.

$a_{1} x$-plane above diagonal; $a_{2} x$-plane below diagonal.

The curve $x=x\left(a_{1} ; \tau\right)$ corresponding to a fixed $\tau>\tau_{c}$ describes discontinuities in $a_{1}(t(\tau), x)$ for which we write $a_{1}(\tau, x)$. When it hits the boundary $\mathrm{S}$ (Styx) at the point $\left(A_{1}^{-}, X\right)$, by Proposition $5, a_{1}(\tau, \cdot)$ jumps discontinuously while $x\left(a_{1} ; \tau\right)$ moves downward through $\mathrm{H}$ to re-emerge at a point $a_{1}=A_{1}^{+}$ lower down. Then it moves upward again (Proposition 5). Somewhat further on it will once again meet the line $x=X$ at $a_{1}=A_{1}$ and cross it. That is where 
the discontinuity in $a_{1}$ ends and $x\left(a_{1} ; \tau\right)$ is (once again) the inverse of $a_{1}(\tau, x)$. Thus we have that $x\left(A_{1}^{-}\right)=x\left(A_{1}\right)=X$. This means that $a_{1}(\tau, X)=A_{1}^{-}$and $a_{1}(\tau, X)=A_{1}$. But $A_{1}^{-}<A_{1}$. Thus there are two $\mathrm{C}_{1}$-characteristics passing through the point $(\tau, X)$ one emanating from the point $\left(0, A_{1}^{-}\right)$and the other from the point $\left(0, A_{1}\right)$. The function $u_{1}$ therefore is discontinuous in the point $(t(\tau), X)$ as we can see from $(10)$.

We need to know more about the motion of the points $\left(A_{1}^{-}, S\left(A_{1}^{-}\right)\right)$and $\left(A_{1}^{+}, S\left(A_{1}^{+}\right)\right)$along $\mathrm{S}$ as $\tau$ varies.

Theorem 8. The function $\tau \rightarrow A_{1}^{-}(\tau)$ decreases to $A_{1}^{\dagger}$ and $X(\tau)$ increases without bound as $\tau \rightarrow \infty$. Likewise, $A_{1}^{+}(\tau) \rightarrow \infty$ as $\tau \rightarrow \infty$.

Proof. Differentiation of the relation (55) with respect to $\tau$ yields $-P_{0}^{\prime \prime}\left(A_{1}^{ \pm}\right) \frac{d}{d \tau}\left[A_{1}^{ \pm}(\tau)\right]=Q^{\prime}(\tau)<0$. Also, $A_{1}^{\dagger}<A_{1}^{-}(\tau)<A_{1}^{0}$. From Theorem 7 it now follows that $A_{1}^{-}(\tau)$ decreases. Similarly, $A_{1}^{+}(\tau)$ increases. Thus the limit $a_{1}^{*}=\lim _{\tau \rightarrow \infty} A_{1}^{-}(\tau)$ exists and $a_{1}^{*} \geq A_{1}^{\dagger}$. Suppose that $a_{1}^{*}>A_{1}^{\dagger}$. Then it follows from (55) that $P_{0}^{\prime}\left(a_{1}^{*}\right)=0$ and that $S\left(a_{1}^{*}\right)$ is finite. This contradicts (54). It follows that $X(\tau)=S\left(A_{1}^{-}(\tau)\right) \rightarrow \infty$. The final statement is proved in the same way.

Our next concern is what happens at the point $\left(A_{1}, X\right)$ where $a_{1}$ leaves the discontinuous jump. We note that by the assumptions, the curve $x=x\left(a_{2} ; \tau\right)$ is continuous and increasing. Let us denote by $A_{2}$ the point where this curve intersects the line $x=X$ (Fig. 3). This point exists for, by (52) $x\left(a_{2}\right) \geq$ $a_{2}-\left[\tau_{2} M^{1 / 2}\right] / P_{0}\left(\tau_{2}\right) \rightarrow \infty$ when $a_{2} \rightarrow \infty$.

Theorem 9. $A_{1}(\tau) \rightarrow \infty$ and $A_{2}(\tau) \rightarrow \infty$ as $\tau \rightarrow \infty$.

Proof. Since $A_{2}(\tau)>X(\tau)$ it follows from Theorem 8 that $A_{2}(\tau) \rightarrow \infty$ as $\tau \rightarrow \infty$. For $A_{1}$ we need to argue differently: in fact, from Theorem 8 we see that $A_{1}(\tau)>A_{1}^{+}(\tau) \rightarrow \infty$ as $\tau \rightarrow \infty$.

\section{Shock discontinuities}

We continue with the hypotheses of Sect.9. From (3) and (5) we find, that $1+p(t, x)=\exp \left\{\frac{1}{2} q_{0}\left(a_{1}(t, x)\right)\right\} \exp \left\{\frac{1}{2} q_{0}\left(a_{2}(t, x)\right)\right\}=P_{0}\left(a_{1}(t, x)\right) P_{0}\left(a_{2}(t, x)\right)$, having used the definition (48). In the same vein one finds

$$
v(t, x)=\frac{1}{2}\left[q_{0}\left(a_{1}(t, x)\right)-q_{0}\left(a_{2}(t, x)\right)\right]=\ln \left[\frac{P_{0}\left(a_{1}(t, x)\right)}{P_{0}\left(a_{2}(t, x)\right)}\right] .
$$

Thus discontinuities in $a_{1}$ transfer to discontinuities in $p$ and $v$. Indeed, jumps in $p$ and $v$, denoted by brackets, can occur at $x=X(\tau)$ and are (for $\tau>\tau_{c}$ ) 
given by

$$
\begin{aligned}
& {[p](\tau)=\left\{P_{0}\left(A_{1}^{-}(\tau)\right)-P_{0}\left(A_{1}(\tau)\right)\right\} P_{0}\left(A_{2}(\tau)\right)} \\
& {[v](\tau)=\frac{1}{2}\left[q_{0}\left(A_{1}^{-}(\tau)\right)-q_{0}\left(A_{1}(\tau)\right)\right] .}
\end{aligned}
$$

Let $m=\lim _{x \rightarrow \infty} P_{0}(x)$. We say that the constraint is challenged if $m=0$. If $m>0$ the constraint is not challenged. From (58) and (59) we obtain:

Theorem 10. If the constraint is not challenged, $[p](\tau) \rightarrow\left\{P_{0}\left(A_{1}^{\dagger}\right)-m\right\} m$ and $[v](\tau) \rightarrow \frac{1}{2}\left\{q_{0}\left(A_{1}^{\dagger}\right)-\ln (m)\right\}$ as $\tau \rightarrow \infty$. If the constraint is challenged, $[p](\tau) \rightarrow 0$ and $[v](\tau) \rightarrow \infty$ when $\tau \rightarrow \infty$.

We are thus confronted by the conclusion that under a challenge to the constraint the "pressure shock" decays to zero while the "velocity shock" gets completely out of hand. This may be understood in the following way: If the initial pressure is low far away, it will tend to distribute evenly while the gas, initially at rest will rush towards places where the pressure is low, impeded by a low propagation speed. There is more to it. Under the hypotheses of Theorem 2 we see from (56) and (57) that for fixed $\tau>\tau_{c}$ and $x \gg X(\tau)$, $1+p(t, x) \sim P_{0}^{2}(x)$ and $v(t, x) \sim 0$. Thus, beyond the shock everything is quite calm. The confluence of space-varying curves in Fig. 3 illustrates this observation and the theorem involved.

Yet, the unboundedness of velocity at the shock is bothersome from an energy point of view. We may, however, consider the power (Watt) experienced by $x$ (reference configuration) at time $t$ defined by

$$
W(t, x):=(1+p) v=P_{0}\left(a_{2}\right) \cdot P_{0}\left(a_{1}\right) \ln P_{0}\left(a_{1}\right)-P_{0}\left(a_{1}\right) \cdot P_{0}\left(a_{2}\right) \ln P_{0}\left(a_{2}\right) .
$$

At $x=X, a_{2}=A_{2}(\tau)$ Theorem 9 shows that $W(t(\tau), X) \rightarrow 0$ as $\tau \rightarrow \infty$. Thus the logarithmic growth of the velocity shock is countered by the rapid drop of pressure.

\section{Decay of pressure and power}

In the previous section we demonstrated the decay of pressure at the shock front when the constraint is challenged. This may also be true for "particles" not in shock. We need to consider the dependence of $a_{2}(t, x)$ on $t$ when $x$ is fixed. In view of Theorem 3 , we only need to consider the dependence of $a_{2}$ on $\tau_{2}$ as given by (52) in the form

$$
a_{2}-\left[\frac{\tau_{2}}{P_{0}\left(\tau_{2}\right)}\right] P_{0}\left(a_{2}\right)=x .
$$

Let $a_{2}\left(\tau_{2} ; x\right)$ denote the solution of $(61)$.

Proposition 11. If $x>A_{1}^{\dagger}$ the function $\tau_{2} \rightarrow a_{2}\left(\tau_{2} ; x\right) ; \tau_{2}>A_{1}^{\dagger}$, is an increasing function of $\tau_{2}$. If the initial pressure $p_{0}$ is non-increasing, this is true for arbitrary (fixed) $x$ and $\tau_{2}>0$. 
Proof. By (11), $a_{2}\left(\tau_{2} ; x\right)>x>A_{1}^{\dagger}$ and hence $P_{0}^{\prime}\left(a_{2}(\tau ; x)\right) \leq 0$. Also, since $\tau_{2} \in I, P_{0}\left(\tau_{2}\right)$ cannot increase. If $p_{0}^{\prime} \leq 0$ everywhere, the same is true for $P_{0}^{\prime}$ and then the first conclusion remains valid.

Let $Q_{2}\left(\tau_{2}\right):=\tau_{2} / P_{0}\left(\tau_{2}\right)$. Since $P_{0}$ is decreasing, $Q_{2}$ is increasing. Differentiation of (11) with respect to $\tau_{2}$ ( $x$ fixed) yields after some re-arrangement

$$
\left[1-Q_{2}\left(\tau_{2}\right) P_{0}^{\prime}\left(a_{2} ; x\right)\right] \frac{d a_{2}}{d \tau_{2}}=Q_{2}^{\prime}\left(\tau_{2}\right) P_{0}\left(a_{2}\right) .
$$

The required assertion now follows in any one of the two situations.

Theorem 12. Suppose that the constraint is challenged. For fixed $x>A_{1}^{\dagger}$, $1+p(t(\tau), x) \rightarrow 0$ when $\tau \rightarrow \infty$. If $p_{0}$ is non-increasing, this is true for arbitrary $x$. In either case the power $W(t(\tau), x) \rightarrow 0$ as $\tau \rightarrow \infty$.

Proof. We first show that $a_{2}\left(x ; \tau_{2}\right) \rightarrow \infty$ when $\tau_{2} \rightarrow \infty$. For this we re-write (11) as follows:

$$
\frac{x}{Q_{2}\left(\tau_{2}\right)}=\frac{a_{2}}{Q_{2}\left(\tau_{2}\right)}-P_{0}\left(a_{2}\right),
$$

Suppose the increasing function $a_{2}\left(\tau_{2}\right)$ has a finite limit $a_{2}^{*}$ then, since $Q_{2}\left(\tau_{2}\right) \rightarrow$ $\infty$, the left and first term on the right of (62) tend to zero as $\tau_{2} \rightarrow \infty$. Hence $P_{0}\left(a_{2}^{*}\right)=0$ and that cannot be. It follows from (56) and (60) that $1+p(t(\tau), x)$ and $W(t(\tau), x)$ tend to zero as $\tau \rightarrow \infty$.

\section{Evolution of states}

We are now able to illustrate the results of Sections $9-11$ by calculating the state of pressure and velocity at different times. The key to such calculations is the expressions (11) and the modus operandi is to calculate, for a fixed time (represented by $\tau$ ) over a range of values of $x$, the inverse characteristics $a_{1}(\tau, x)$, $a_{2}(\tau, x)$. From there the velocity $v(t, x)$ and pressure $p(t, x)$ can be evaluated. The results for Ex. 1 are shown in Fig. 4.

The development of discontinuities is clearly seen. An interesting feature is the development of motion in a medium initially at rest. So is the decline of the initial pressure and the apparent confinement of both velocity and pressure in the downstream direction. The effect is the result of the very rapid decay downstream from the shock. It must be kept in mind, though, that the shock occurs at $x=X(\tau)$, a point that keeps going forward in time. The shock curve $x=X(\tau)$ increases quite rapidly. In fact, from (50) and Theorem 9 we have the asymptotic behaviour

$$
X(\tau) \sim A_{1}^{\dagger}+P_{0}\left(A_{1}^{\dagger}\right) \frac{\tau}{P_{0}(-\tau)} \quad \text { as } \tau \rightarrow \infty .
$$

In Ex. 1 equation $(63)$ is $X(\tau) \sim \tau$. 

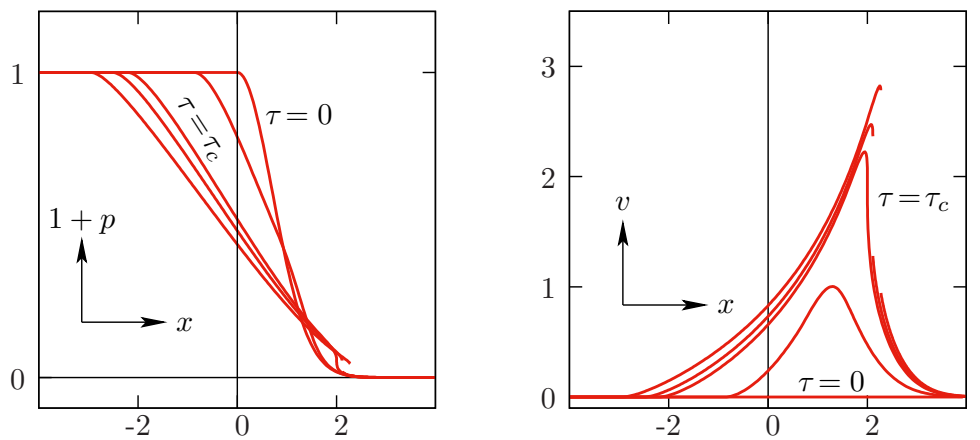

Figure 4: Evolution of pressure (left) and velocity (right): Ex. 1
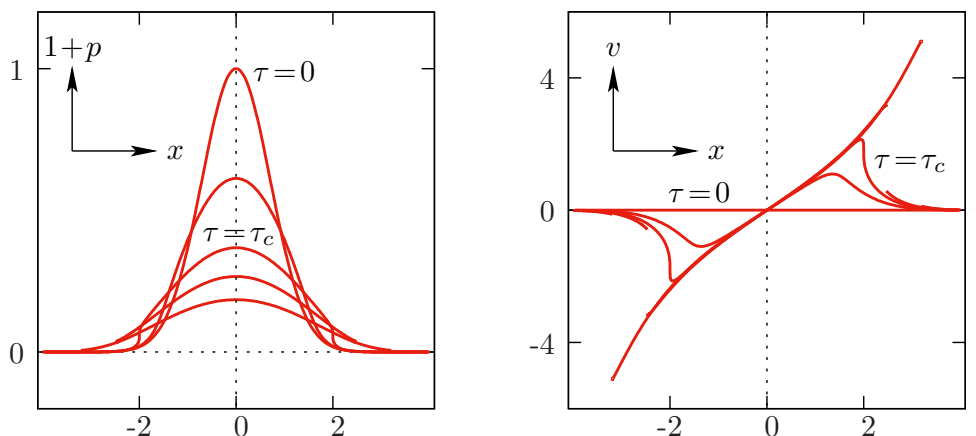

Figure 5: Evolution of pressure (left) and velocity (right): Ex. 2

In Fig. 5 we show the evolution of states for Ex. 2 where $1+p_{0}(x)=$ $\exp \left\{-x^{2}\right\}$ also has an increasing part and the constraint is challenged at both ends $( \pm \infty)$. Since in this case $p_{0}$ is an even function, Theorem 1 lessens the burden of calculation. One only has to calculate the profiles for $x \geq 0$ and the reflect according to (25). For this particular choice $\tau_{c}=1$ although the curve of discontinuity is the same as for Ex. 1. The equation (63) is $X(\tau) \sim \tau \exp \left\{\tau^{2} / 2\right\}$ which is phenomenal. The (mathematical) phenomena discussed in the previous section are perhaps more vividly demonstrated in Fig. 5.

It should be noted that in the model-examples, time $t$ increases very quickly with (compressed) time $\tau$ so that the calculated shock phenomena correspond to comparatively large times.

\section{Linearizations}

It is also of interest to compare the outcomes above with those for linearizations when it is assumed that $|p(t, x)| \ll 1$. This kind of linearization suggests 
that the Jacobian $J$ (Sect. 1) is close to unity which means that the gas is virtually incompressible.

Two instances come to mind. The first is to collapse the equations (1) into the simple linear symmetric hyperbolic system

$$
\left\{\begin{array}{l}
v_{t}+p_{x}=0 \\
p_{t}+v_{x}=0
\end{array}\right.
$$

This system can be easily solved. Under the initial conditions considered here the solution is $v(t, x)=\frac{1}{2}\left[p_{0}(x-t)-p_{0}(x+t)\right] ; 1+p(t, x)=\frac{1}{2}\left[\left(1+p_{0}(x-t)\right)+\right.$ $\left.\left(1+p_{0}(x+t)\right)\right]$. Thus the pressure simply divides into two identical parts, the one going down and the other upstream. Likewise, the velocity evolves into an up and anti-phased downstream part. In this case pressure does not decay and velocity behaves as introductory texts in physics predict.

The second instance is an attempt to accommodate the constraint by linearization of the system (4) with the result

$$
\left\{\begin{array}{l}
v_{t}+q_{x}=0 \\
q_{t}+v_{x}=0 .
\end{array}\right.
$$

The solution is obtained with equal ease. Indeed, $v(t, x)=\frac{1}{2}\left[q_{0}(x-t)-\right.$ $\left.\left.q_{0}(x+t)\right] ; q(t, x)=\frac{1}{2}\left[\left(q_{0}(x-t)\right)+q_{0}(x+t)\right)\right]$. Of course the solution of $(65)$ has to be transformed back to pressure by (3) with the result that $1+p(t, x)=$ $\left.\exp \left\{\frac{1}{2}\left[\left(q_{0}(x-t)\right)+q_{0}(x+t)\right)\right]\right\}=P_{0}(x-t) P_{0}(x+t)$. This is quite different from the first linearization. Indeed, decay of pressure is evident as is the velocity getting out of hand.

Nonlinear behaviour is totally different. In linear systems the characteristics are not determined by the initial state and shock discontinuities cannot occur. Yet, at least one linearization exhibits decay of pressure.

It may also be prudent to compare the system (1) and its linearization (64) from the point of view of symmetry. In the original system, the unknown functions $v$ and $p$ cannot be interchanged, while in the linearized system, they can. Symmetry in the linear system is typical of classical wave phenomena. The a-symmetry of the nonlinear system shows why the constraint can only be challenged by the initial pressure.

The linearized system (64) leads (under some assumptions of differentiability) to the wave equation (Friedrichs [6]) which is still the basis of much of acoustic engineering, loudspeaker design and heuristic reasoning.

\section{Some words and no symbols}

We have seen that when the input pressure decreases, shock discontinuities in both pressure and velocity will develop and pressure will decay. If the constraint is challenged, pressure will decay towards the allowed lower limit and velocity will increase unboundedly with time at the shock. This seems to be un-physical. 
The mathematical constraint we impose may be at the core. Physically the constraint should be stricter in the sense that a positive lower bound would be more appropriate. In that case, we found, that velocity will indeed not get out of hand.

The sudden drop of velocity ahead of the shock may be seen in the pressuredependent propagation speed. When this quantity is near zero, disturbances will find it hard to propagate, which heuristically accounts for the violent shock.

Challenges to the constraint as studied here, also have a mathematical side. It is the constraint that makes the system of differential equations hyperbolic. When the constraint is challenged, hyperbolicity is challenged in the sense that two crucial eigenvectors become almost linearly dependent. One should also observe that the Boyle-Mariotte relation implies that when the constraint is challenged the deformation becomes excessive which is another way of understanding the violent behaviour at shocks.

\section{References}

[1] C.M. Dafermos. Hyperbolic Conservation Laws in Continuum Physics. Number 325 in Grundlehren der mathematischen Wissenschaften. SpringerVerlag, Berlin Heidelberg New York, second edition, 2005.

[2] S. Earnshaw. On the mathematical theory of sound. Proc. Roy. Soc. London, 9:590-591, 1858.

[3] R. Courant and K.O. Friedrichs. Supersonic Flow and Shock Waves. Interscience Publishers, New York, 1948.

[4] A. Jeffrey. The evolution of discontinuities in solutions of homogeneous nonlinear hyperbolic equations having smooth initial data. Indiana Univ. Math. J. (formerly, J. Math. Mech.), 17:331-352, 1967.

[5] A. Jeffrey. Quasilinear hyperbolic systems and waves. Pitman Publishing, London-San Francisco-Melbourne, 1976.

[6] K.O. Friedrichs. Symmetric positive linear differential equations. Comm. Pure Appl. Math., 11:333-418, 1958.

[7] P.D. Lax. The formation and decay of shock waves. Amer. Math. Monthly, $79: 227-241,1972$.

[8] P.D. Lax. Hyperbolic systems of conservation laws and the mathematical theory of shock waves. SIAM Regional Conference Series in Applied Mathematics, 11:1-48, 1973.

[9] N. Sauer. An excursion into classical nonlinear acoustics. Int. J. Eng. Sci., 48:670-684, 2010.

[10] N. Sauer. The dynamic piston problem in classical nonlinear acoustics. Math. Models and Meth. Appl. Sci., 21:149-167, 2011. 
[11] T.Y. Thomas. The growth and decay of sonic discontinuities in ideal gases. Indiana Univ. Math. J. (formerly, J. Math. Mech.), 6:455-469, 1957. 